\title{
Accurate detection of spread-spectrum modulated signals in reverberant underwater environments
}

\author{
Joaquín Aparicio ${ }^{\mathrm{a}, *}$, Ana Jiménez ${ }^{\mathrm{a}}$, Fernando J. Álvarez ${ }^{\mathrm{b}}$, Jesús Ureña ${ }^{\mathrm{a}}$, Carlos De Marziani ${ }^{\mathrm{c}}$, \\ Daniel de Diego ${ }^{a}$, Nuno Cruz ${ }^{\mathrm{d}}$, Helder Campos ${ }^{\mathrm{d}}$ \\ ${ }^{a}$ Dept. of Electronics, University of Alcalá, Campus Universitario, Ctra. Madrid-Barcelona, Km. 33,600, 28871 Alcalá de Henares, Spain \\ ${ }^{\mathrm{b}}$ Dept. of Electrical Engineering, Electronics and Automatics, University of Extremadura, Facultad de Ciencias, Edificio de Fisica, 06006 Badajoz, Spain

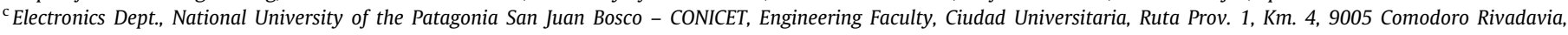 \\ Argentina \\ ${ }^{\mathrm{d}}$ Dept. of Electrical and Computer Engineering, INESC TEC (Institute for Systems and Computer Engineering of Porto), Faculty of Engineering, University of Porto, Rua Dr. Roberto \\ Frias, 4200-465 Porto, Portugal
}

\section{A R T I C L E I N F O}

\section{Article history:}

Received 19 March 2014

Received in revised form 18 July 2014

Accepted 6 August 2014

\section{Keywords:}

Multipath environments

Underwater acoustic propagation model

Spread-spectrum signals

\begin{abstract}
A B S T R A C T
In those positioning systems based on the detection of acoustic signals, an accurate detection of the arrival times is crucial for a correct estimation of the distance between nodes, and therefore, for the precise estimation of the node that wants to be located. In order to obtain this arrival time more accurately, acoustic signals can be coded using pseudorandom noise, but these coded signals are still affected by underwater channel phenomena. In this work, the detection of spread-spectrum modulated signals is analyzed in underwater environments that are highly affected by multipath and reverberation. A spread-spectrum signal, which consist of a modulated Kasami code, has been emitted through two different pools, reaching a receiver where it has been captured after following several line-of-sight and nonline-of-sight paths. Then, a correlation process has been performed offline to provide information about the arrival times (times-of-flight) that form the multipath structure. These times-of-flight are compared with those provided by an underwater acoustic propagation model, in order to test the performance of this model and its capacity to predict the outcome of signal detection in underwater environments with a strong multipath and reverberation component. That way, the validated propagation model could be later used in future studies to predict the detection of spread-spectrum signals and the performance of systems that use them in these adverse environments.
\end{abstract}

(c) 2014 Elsevier Ltd. All rights reserved.

\section{Introduction}

Underwater medium is a challenging environment that shows a high variability, both spatial and temporal. Acoustic signals that propagate through underwater channels are usually affected by multipath, generating at the receiver end several copies of the emitted signal which can worsen the functioning of underwater systems due to the interference between the different symbols (Inter-symbol interference, ISI).

In order to reduce the impact of this effect and also to improve the process gain against noise, the acoustic signal can be coded by means of spread-spectrum techniques (Direct-Sequence CodeDivision Multiple Access, DS-CDMA). These techniques are not as sensitive as Time-Division Multiple-Access techniques (TDMA)

\footnotetext{
* Corresponding author.

E-mail address: joaquin.aparicio@depeca.uah.es (J. Aparicio).
}

with respect to long and variable delay times that are common in this medium, and they are more robust to Doppler effects than Frequency-Division Multiple Access techniques (FDMA). Additionally, CDMA techniques do not require an equalization stage before the detection of the received signal, although it is sometimes used to improve the results.

For all these reasons, CDMA has become a useful technique against multipath, reverberation and fading since the beginning of the 1990 decade [1,2]. They also provide more precise estimations of the distance between two nodes, which is crucial in sonar or positioning systems [3]. On the other hand, the use of DS-CDMA techniques implies the use of a larger bandwidth in the emitted signal, as well as a reduction in the bit rate, which could be an important drawback in some systems [4].

Through all these years, different works have reported the use of coded signals mostly in communications experiments, using Gold codes with a Rake receiver in an underwater modem [5], or 
comparing the results of OFDM modulated Gold codes (Orthogonal Frequency Divison Multiplex) and an equalizer at the receiver, with an DSSS modulation scheme [6]. More recent studies have used msequences to obtain more process gain against noise using a PPC receiver (Passive Phase Conjugation) [7].

Additionally, testing underwater acoustic systems can be a very complex task, with a high consumption of temporal and economic resources. In order to perform preliminary studies of such systems without the restriction of location or environmental conditions, the use of propagation models have become a powerful technique. Up to date, there are different mathematical techniques that can be used to simulate the acoustic propagation in underwater environments, such as ray-tracing, normal-mode models and the parabolic equation.

Among all of them, ray-tracing is the best one for highfrequency modeling [8], which is particularly useful for the study of spread-spectrum techniques, in which the carrier frequency is in the order of the kiloHertz or greater. Ray-tracing provides an intuitive approximation to the acoustic propagation and multipath, the directionality of the sources can be defined, and they can handle bathymetry profiles and range-dependent sound speed profiles. On the other hand, they are not recommended to study low-frequency propagation or bottom interactions [9]. Several ray-tracing software applications have been developed through these decades, each of them applicable to a certain kind of environment and with some restrictions. Some of the most popular models are GRAB [10], RAY [11] or Bellhop [12], but different authors propose their own models to fit their specific needs. For example, the model by Niese and Lützen [13] uses a Monte Carlo method to predict signal transmission conditions and fading for turbulent shallow water environments, and the model by Chitre [14], valid for very shallow water environments, includes time-varying statistical effects and non-Gaussian noise.

In this work, an experimental study of the detection of coded signal in underwater environments highly affected by multipath is presented. One of the objectives is to test the correct detection of a BPSK modulated Kasami code in these adverse environments using only a matched-filter, avoiding the use of more complex receivers. The other objective is the comparison of these results with those provided with an underwater acoustic propagation model, which would demonstrate the feasibility of this model to predict signal detections in different environments.

The rest of the paper is organized as follows. In Section 2 an explanation of the model is presented. Section 3 shows the results for the signal detection in two different pools, comparing the results from the experiment and the model predictions. Finally, Section 4 summarizes the conclusions and future work.

\section{The underwater acoustic propagation model}

In this section, the propagation model is described. The model has been programmed in a Matlab environment, and considers different phenomena such as geometrical spreading, absorption and rebound losses, as well as Doppler-spread caused by windgenerated waves in the surface, when present. The model is valid for shallow and deep water environments as it can handle customizable sound speed profiles. Also, it can use a bathymetry profile with an arbitrary shape. Other ray-tracing programs were not well suited for this study, as they use a set of equations whose range of validity were not appropriate, they do not include some effects that could be important to consider in signal-processing systems, or they are limited to specific environments.

Fig. 1 shows the block diagram of the programmed algorithm computations. This algorithm needs some environmental inputs such as water and bottom densities, sound speed at bottom

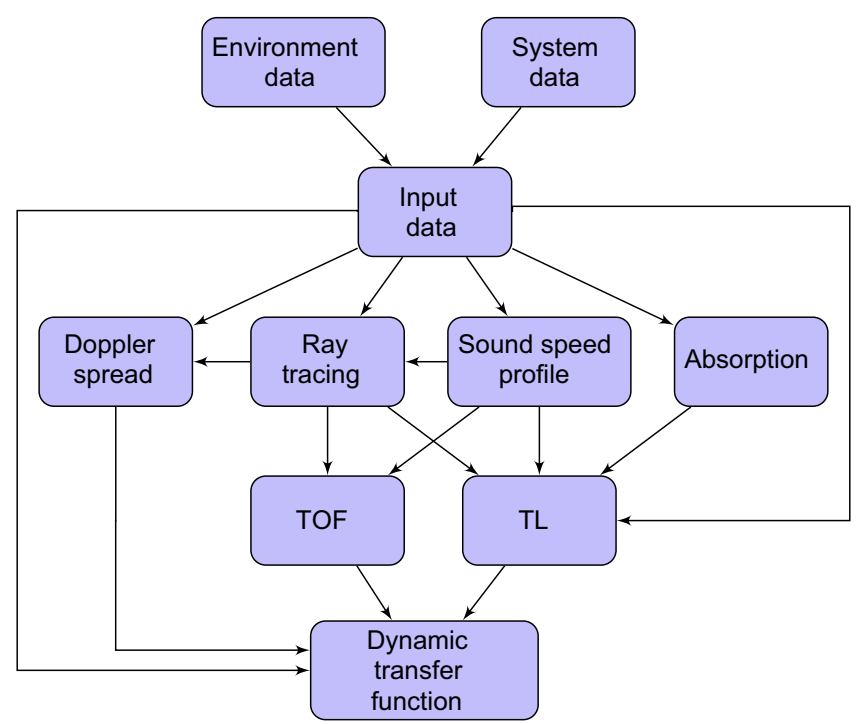

Fig. 1. Block diagram of the propagation model.

material, water temperature (or the sound speed profile directly), salinity, $\mathrm{pH}$, latitude, the signal-to-noise ratio (SNR) considering Gaussian noise, the bathymetry profile and the wind speed at the surface. It also needs some system data, such as the positions of the transducers and their depths, their aperture angle, signal frequency and sampling frequency.

With all these data, the first step is to calculate the sound speed profile and to perform a ray-tracing in the defined environment. This ray-tracing is two-dimensional, in the vertical plane that connects the emitter with the receiver. With this information and the previous data, the propagation times can be obtained. The absorption coefficient is calculated from the input data, whereas the transmission loss $(T L)$ equations use this coefficient and the information obtained with the ray-tracing to calculate the loss values for all the rays. Doppler spread is calculated for the rays that bounce off the sea surface, and lastly a dynamic transfer function is computed by using the internal Matlab function rayleighchan. This function takes as inputs the sampling frequency and transmission loss, Doppler spread and delay times for the eigenrays, and it provides a received signal with Rayleigh fading amplitude and random phase shifts for the surface reflected signals.

First, an explanation of how the ray-tracing technique has been implemented will be presented, and next, the fundamental phenomena of underwater acoustic propagation that are included in the model, such as sound speed, transmission loss and the dynamic effect of wind-generated waves, will be revised.

\subsection{Ray-tracing technique}

This propagation model does not solve the differential equations of the ray-tracing technique. Instead, it uses a geometrical approach. The water column is assumed to be stratified in layers of a custom depth. In each of these layers, sound speed is considered to be constant, but its value can change from one layer to the next. Thus, a ray path will follow a straight line within one layer, but when it hits the next one, the sound speed value changes and so does the angle of the ray path, according to Snell's Law. This change in the angle causes the curvature of the ray as a consequence of the sound speed profile. Large values for the layers depth (in the order of meters) will result in large sections of water where the sound speed value is not changing as it should. This effect will cause an inaccuracy in the estimation of the ray paths. However, small values for the layers depth (in the order of millimeters) will 
result in higher computation times, as the model needs to calculate the ray paths in more sections. Thus, the optimal value is a tradeoff between accuracy and computational time, where a value of $10 \mathrm{~cm}$ is usually employed in the model. Naturally, the computational cost of the model will also increase with the size (depth and range) of the considered environment.

A large number of rays are launched at the emitter between custom angles. The number of rays depends on the environment. For environments with a strong multipath component due to long propagation (such as hundreds of meters) in shallow water scenarios with a bottom depth of few meters, the model needs a large number of rays (typically between 600 and 700) in order to describe all the possible paths. If the multipath component is not that strong, fewer rays can be used. These rays will propagate through the medium, bouncing off the sea-surface and the bottom; the sea-surface is considered to be flat for computing the raytracing, although the movement of the surface is taken into account later for the Doppler spread and fading of the reflected waves. A bathymetry profile can be included, as a depth value of the bottom every certain meters. The shorter the distances between the bottom depth values, the more accurate the bathymetry profile is, but at the expense of increasing the computational cost. The rays will reach the receiver end at certain times. The propagation times for these rays are computed in each layer taking into account the sound speed value in the layer, and then, these times are accumulated.

Once all the rays have reached the receiver end, the eigenrays are detected. The model identifies eigenrays by means of ray beams, where one ray beam is formed by two contiguous rays with the same properties of rebounds at the sea surface and the bottom. If the receiver is placed inside a ray beam, the model detects and eigenray, and it interpolates the propagation time and transmission loss that would have a ray hitting the exact position of the receiver, considering the values of the two rays of the beam. This situation is represented in Fig. 2 with the rays in solid lines, whereas the receiver is represented as a triangle. If the receiver is placed outside a ray beam, like the one in dashed lines, no eigenray is detected by the model.

\subsection{Sound speed}

The theoretical equation for the sound speed in water, $c$, is shown in (1), where $\gamma$ is the adiabatic index, $B_{T}$ is the isothermal bulk modulus and $\rho_{0}$ is the equilibrium density [15]:

$c^{2}=\gamma \frac{B_{T}}{\rho_{0}}$

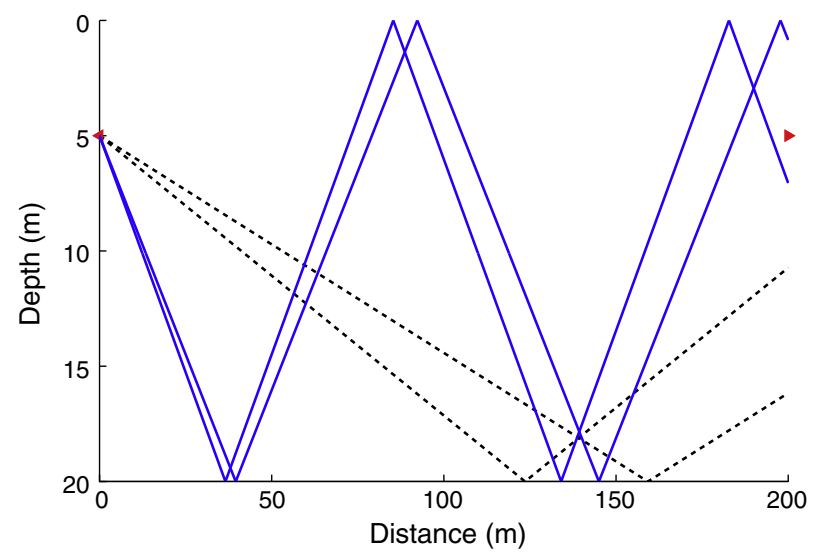

Fig. 2. Eigenray detection.
However, this equation is not implemented in propagation models, because these three variables dependence on temperature and water depth is not easy to predict. Thus, several semiempirical formulas have been proposed through the last decades, in order to compute sound speed based on temperature $(T)$, pressure $(P)$ and salinity $(S)$. In this work, the formula by Chen and Millero [16] (2) is used to obtain the sound speed, because of its wide range of valid inputs for these variables. This equation can be used for water temperature between $0{ }^{\circ} \mathrm{C}$ and $40^{\circ} \mathrm{C}$, pressure between 0 bar and 1000 bar and salinity between 0 ppt and 40 ppt. With these values, almost all kinds of scenarios can be covered using the Chen-Millero equation. The values for the functions $C_{w}, A, B$ and $D$ can be consulted in [16].

$$
\begin{aligned}
c(S, T, P)= & C_{w}(T, P)+A(T, P) S+B(T, P) S^{3 / 2} \\
& +D(T, P) S^{2}\left(\mathrm{~m} \mathrm{~s}^{-1}\right)
\end{aligned}
$$

Sound speed is computed in each layer, knowing the temperature profile and salinity for the water column. Pressure is obtained using the Leroy-Parthiot equation [17], which provides a depth to pressure conversion. A sound speed profile is then obtained, and this way the model can be applied both in shallow and deep waters. No range-dependence is considered yet in the sound speed profile.

\subsection{Transmission loss}

The propagation of acoustic waves in water has three main causes of energy loss: geometrical spreading, water absorption and rebounds in the sea-surface and the bottom. This transmission loss is computed for each ray, taking into account the followed path.

The expansion of the acoustic wave in the medium causes the geometrical spreading, $T L_{\text {geo }}$. There are two types of spreading, depending on the geometry of the channel: cylindrical and spherical spreading. The acoustic waves tends to propagate spherically, but if these waves are too constrained, like in a very shallow water environment, where the range between emitter and receiver are several orders of magnitude higher than depth, then it can be considered that they propagate cylindrically, losing less energy in the process. Eq. (3) shows the geometrical spreading loss, where $k$ is a constant, whose value is 10 for cylindrical spreading, and 20 for spherical spreading [18], and $r$ is the distance traveled by the ray in meters.

$T L_{\text {geo }}=k \log r \quad(\mathrm{~dB})$

The absorption term, $T L_{a b s}$, takes into account the part of the energy of the wave that is transferred to the medium as heat. This energy loss depends on the total distance traveled by the ray and the absorption coefficient, $\alpha$, following (4):

$T L_{a b s}=\alpha r 10^{-3} \quad(\mathrm{~dB})$

The absorption coefficient can be computed by means of different semiempirical equations. In this work the one by Francois and Garrison has been used, as it is shown in (5) $[19,20]$, because of the range of valid inputs frequencies, ranging from $200 \mathrm{~Hz}$ to $1 \mathrm{MHz}$. This equation is especially useful when working with highfrequency signals (tens of $\mathrm{kHz}$ ), such as the ones used in this work, as opposed to Thorp equation, that is employed in other models and it is recommended only to few $\mathrm{kHz}$ [21].

$\alpha=\frac{A_{1} P_{1} f_{1} f^{2}}{f^{2}+f_{1}^{2}}+\frac{A_{2} P_{2} f_{2} f^{2}}{f^{2}+f_{2}^{2}}+A_{3} P_{3} f^{2} \quad\left(\mathrm{~dB} \mathrm{~km}^{-1}\right)$ 
In this equation, $A_{i}$ and $P_{i}$ are functions that can depend on the sound speed profile, temperature, salinity, depth and $\mathrm{pH}$. Their value can be consulted at [20]. Parameters $f_{1}, f_{2}$ are the relaxation frequencies of boric acid and magnesium sulfate, respectively, and $f$ is the wave frequency, all of them given in $\mathrm{kHz}$. Given the dependence on the sound speed profile, the absorption coefficient is computed in all the water column, and the correct value is taken to compute the absorption loss depending on the depth of the ray in that moment, that is, in which layer the ray is. Thus, absorption loss is computed in each step from one layer to another for each ray, and it is accumulated.

The bottom loss, $T L_{b o t}$, can be computed in the model by two different ways. One of them, by considering a constant bottom loss per rebound, defined by the user. The other one, by using the Rayleigh model [18], given by (6), which is one of the easiest ways to compute the bottom loss in practice:

$T L_{b o t}=10 \log \left[\frac{q \sin \theta-\left(n^{2}-\cos ^{2} \theta\right)^{1 / 2}}{q \sin \theta+\left(n^{2}-\cos ^{2} \theta\right)^{1 / 2}}\right]^{2}$

where $q=\rho_{b} / \rho_{w}$ and $n=c_{w} / c_{b} . \rho_{b}$ is the bottom density and $\rho_{w}$ is the water density, both of them in $\mathrm{kg} \mathrm{m}^{-3} . c_{w}$ is the sound speed in water and $c_{b}$ is the sound speed in bottom material, both of them in $\mathrm{m} \mathrm{s}^{-1}$. Lastly, $\theta$ is the angle of incidence in the bottom.

The last contribution to energy loss are the rebounds at the sea surface, $T L_{\text {sur }}$. One of the simplest equations to calculate the energy loss at the surface is the Beckmann-Spizzichino equation. In this work, this equation has been implemented in the form given by Coates [22], which is shown in (7):

$T L_{\text {sur }}=10 \log \left(\frac{1+\left(f / b_{1}\right)^{2}}{1+\left(f / b_{2}\right)^{2}}\right)-\left(1+\frac{90-w}{60}\right)\left(\frac{\theta_{s}}{30}\right)^{2}$

where $f$ is the signal frequency in $\mathrm{kHz}, w$ is the wind speed in knots, $\theta_{s}$ is the angle of incidence at the surface in degrees, $b_{1}=\sqrt{10} b_{2}$ and $b_{2}=378 w^{-2}$.

The total transmission loss for each ray can be then obtained by adding all the previous terms, as shown in (8), where $n_{\text {bot }}, n_{\text {sur }}$ are the total number of rebounds suffered by the ray at the bottom and the surface, respectively, and the minus sign corrects the positive value of these magnitudes, as the rayleighchan function needs a negative value for the transmission loss.

$T L=-T L_{g e o}-T L_{a b s}+n_{b o t} T L_{b o t}+n_{\text {sur }} T L_{\text {sur }}$

Considering the total transmission loss for each eigenray $T L_{i}$, the attenuation coefficient $A_{i}$ for that particular eigenray can be obtained following (9). That way, the received signal $Y_{i}$ obtained from the emitted signal $X$, can be calculated as $Y_{i}=A_{i} \cdot X$. This process is applied to each eigenray, and the total received signal is obtained by adding all the contributions.

$A_{i}=10^{T L_{i} / 20}$

\subsection{The dynamic effect of wind-generated waves}

Although the sea surface is assumed to be flat for computing the ray paths, the effect of wind-generated waves is considered in the model. These waves will cause a movement of the reflection point of the surface-reflected rays, and so a Doppler spread $B$ is added to these signals. The Doppler spread is given by (10) [23]

$B=0.0175\left(\frac{f}{c}\right) w^{3 / 2} \cos \theta_{s}$

In this equation $f$ is the signal frequency in $\mathrm{Hz}, c$ the sound speed in $\mathrm{m} \mathrm{s}^{-1}, w$ the wind speed in $\mathrm{m} \mathrm{s}^{-1}$ and $\theta_{s}$ the angle of incidence to the surface. This Doppler spread causes a widening of the signal spectrum, which will worsen the properties of the coded signals. Additionally, the surface-reflected signal will be affected by fading due to the movement of the sea-surface. Thus, this signal will suffer from changes in amplitude and signal phase fluctuations. Considering Doppler spread and fading, a dynamic transfer function is then computed. This function applies a Rayleigh fading in amplitude to the signal, as well as a random phase shift following an uniform distribution, whereas the signal spectrum is spread. If there is no wind speed, the sea-surface can be considered like a perfect reflector, and then the model only considers a phase shift of $180^{\circ}$ in the reflected signal, in addition to energy loss.

\section{Experimental detection of coded signals}

The model has been used to predict the detection of spreadspectrum acoustic signals that propagate in underwater channels with a strong multipath component. Two different tests have been conducted to compare this prediction with real data. These tests provide valuable information on two important issues: on one hand, if the spread-spectrum signals can be detected in these unfavorable conditions for communications, it can be assumed that it will be easier to detect these signals in open environments with less multipath and reverberation; on the other hand, if the model correctly predicts the detection of the signal, it could be reliably used later on to perform preliminary studies of performance of more complex systems in environments that can be affected by multipath as well as by other effects.

The experiments have been performed in two different pools. One of them is placed outdoors in a sports facilities in Alcalá de Henares, Spain (test one), whereas the other one is placed indoors in the Ocean Systems Group facilities at the University of Porto, Portugal (test two). In both cases, one emitter and one receiver were placed at opposite ends of the pools. A spread-spectrum signal is emitted in both tests, which consists in a BPSK modulated Kasami code. When the coded signal is sent, it will arrive at the receiver after following different paths with different times-offlight (TOF). This signal will be recorded. A matched filter will then be employed offline to detect the emitted code as the correlation peaks of the resulting signal, and to obtain the TOF as the difference between the arrival time and the signal duration. The objective of these tests is to identify these arrivals and compare the results for the TOF of the real and simulated signals.

Synchronization is needed between emitter and receiver to obtain these TOF. This synchronization was achieved differently in both experiments. In test one, synchronization was obtained using a radio-frequency link. In test two, it was achieved by connecting both the emitter and receiver to the same FPGA (Field-Programmable Gate Array).

Since the model is two-dimensional, some propagation information is missed from the environment: no rebounds in the lateral walls of the pool are originally included in the simulation, neither are rebounds from the walls placed behind both transducers. However, because of the regular geometry of the pools, it is easy to consider the effect of some of these paths. For both tests the procedure is the same. First, a simulation following the usual vertical propagation plane is performed, and the received signal is stored. Then, a rotation of the propagation plane of $90^{\circ}$ is considered in the simulation, so now the model calculates the propagation in the horizontal plane, as can be seen in Fig. 3. For the simulation of this horizontal propagation plane, the dimensions of the pool are adjusted, as well as the rebound losses, since now both surface and bottom are made of concrete. The received signal is then stored, but the direct path is subtracted, since it is the same than in the previous simulation. Lastly, the rebounds from the walls behind both transducers that follow a straight path between them 


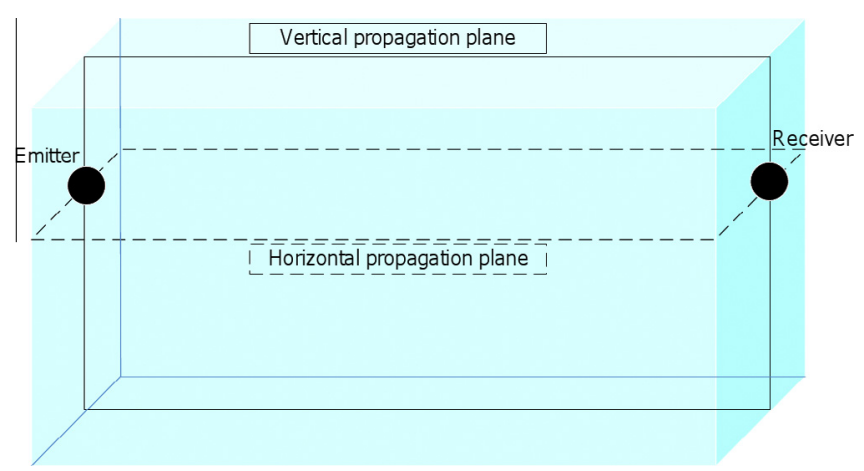

Fig. 3. Propagation planes considered in the simulations.

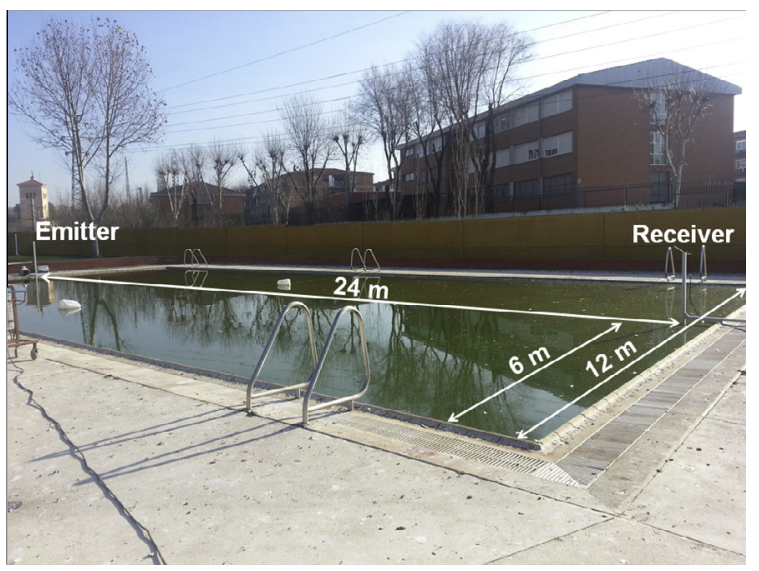

Fig. 4. Set up for test one.

are also included, since the total distance can be computed easily and added to the simulated results. In test one, only the path bouncing off the receiver wall is added, because of the directionality of the emitter. In test two, three paths have been added with these characteristics: one bouncing off the emitter wall, a second one bouncing off the receiver wall, and a third one bouncing off both walls, due to the omnidirectional characteristic of the emitter and the receiver.

In both simulations, a water density of $1000 \mathrm{~kg} \mathrm{~m}^{-3}$ and bottom density of $2300 \mathrm{~kg} \mathrm{~m}^{-3}$ have been considered, since both pools are made of concrete. Thus, sound speed in the bottom material was considered to be $3600 \mathrm{~m} \mathrm{~s}^{-1}$. No wind speed was considered, as in test one there was calm weather with no wind-generated waves, and the pool in test two is placed indoors. A reasonable SNR value has been considered for both simulations (which will be specified later), considering a favorable scenario since they are controlled environments. Needless to say, gathering a priori information about local SNR would be useful for a proper simulation of the propagation in an open environment.

\subsection{Test in pool one}

The pool used for this test has a large size, and additionally, the emitter has a high directionality, avoiding much of the rebounds from the lateral walls of the pool. This configuration represents a scenario with a considerable multipath component, but not extremely severe since the contribution of lateral walls is not very important. The set up and the dimensions of this pool are shown in Fig. 4. Emitter and receiver were placed at $50 \mathrm{~cm}$ depth. Fig. 5(a) and (b) shows an example of ray-tracing using 20 rays per visual clarity for the vertical and horizontal plane, respectively.

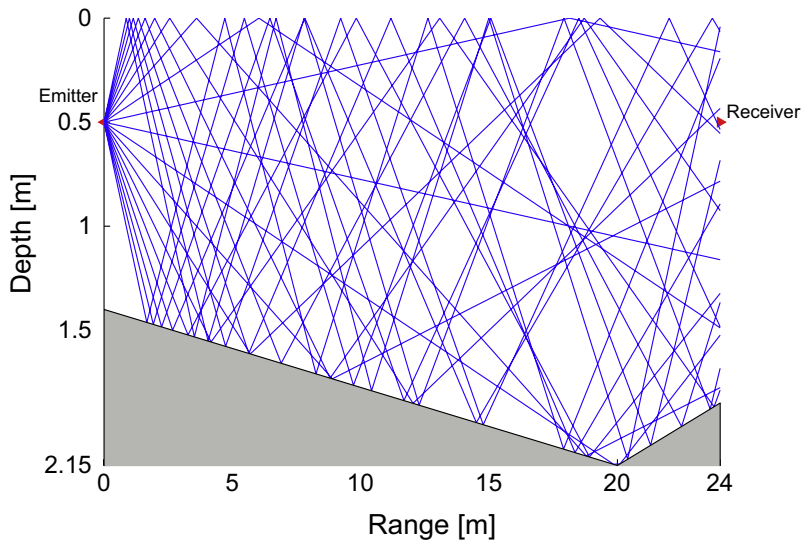

(a) Vertical plane

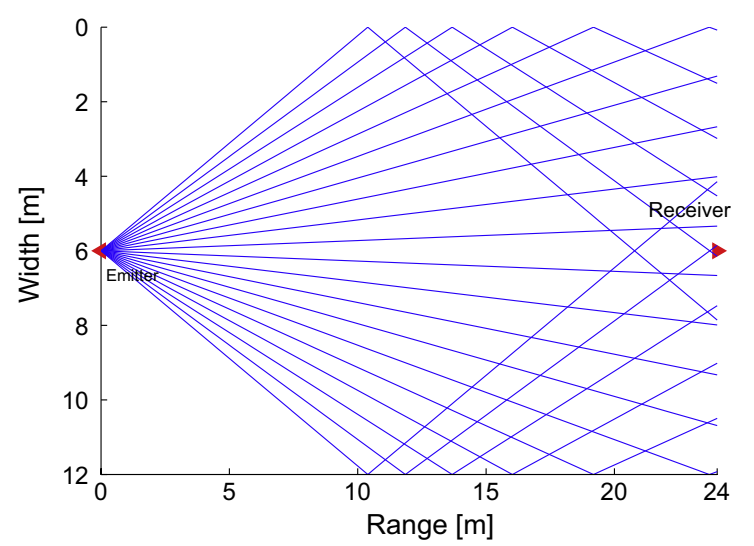

(b) Horizontal plane

Fig. 5. Ray-tracing for: (a) the vertical plane and (b) the horizontal plane in test one.

The bathymetry profile of the pool is also displayed. The bottom depth is $1.40 \mathrm{~m}$ at the receiver, reaches a maximum of $2.15 \mathrm{~m}$ at $20 \mathrm{~m}$ distance, and slightly increases up to $1.85 \mathrm{~m}$ at the receiver's position.

The emitted signal consist of a 63 bits Kasami code [24], BPSK modulated using 2 cycles per symbol for a total duration of approximately $0.6 \mathrm{~ms}$ and a bandwidth of $100 \mathrm{kHz}$, as shown in Fig. 6. The emitter is a high-frequency ProWave 200LM450 transducer with a bandwidth of $25 \mathrm{kHz}$ and a beamwidth of $20^{\circ}$ (horizontal and vertical), considering a signal loss of $6 \mathrm{~dB}$. In this test, the carrier

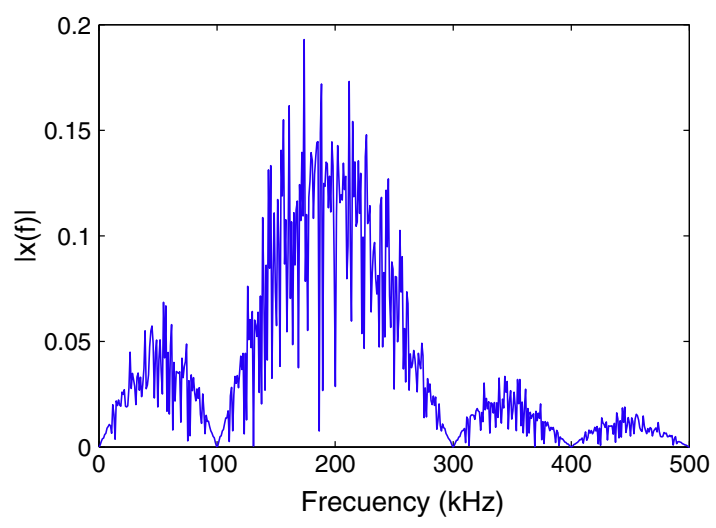

Fig. 6. Amplitude spectrum of the emitted signal in test one. 


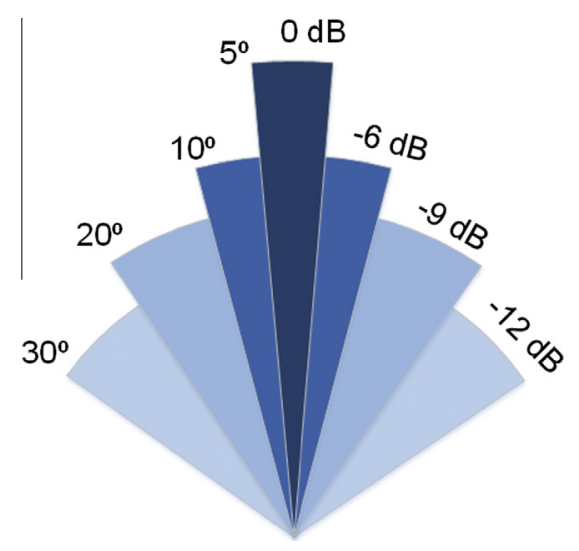

Fig. 7. Approximated beam pattern for the ProWave 200LM450 transducer.

frequency is $200 \mathrm{kHz}$. At the receiver, an omnidirectional Reson TC4034 hydrophone was used to acquire and store the signal in a computer at a sampling frequency of $1 \mathrm{MHz}$.

In order to obtain the simulated signal, the rays were launched between $-30^{\circ}$ and $30^{\circ}$ to replicate the characteristic emission of the ProWave 200LM450. The only information about the beam pattern of this emitter is a signal loss of $6 \mathrm{~dB}$ for an angle greater than $\pm 10^{\circ}$. For that reason, the beam pattern has been approximated as shown in Fig. 7. That way, a signal loss of $6 \mathrm{~dB}$ is considered for a beam of $20^{\circ}$, as specified by the manufacturer, and then there is $3 \mathrm{~dB}$ of extra loss each time the beam gets $20^{\circ}$ wider, in order to simulate a simple energy attenuation model for the emitter.

A value of $0 \mathrm{~m} \mathrm{~s}^{-1}$ for the wind speed, $12 \mathrm{~dB}$ of SNR and a water temperature of $8{ }^{\circ} \mathrm{C}$, which was measured on site and considered constant for all the water column, were used in the simulation. The dimensions have been defined in Fig. 4, and the information of frequency response provided by the transducer manufacturer is also included.

Due to the fact that a directional emitter is used, only the signal bouncing off the receiver wall is added to the main plane and the lateral plane in the simulated signal. The same Kasami signal that is used in the test pool is used in the propagation model. Since the signal is normalized at the receiver to store it in a computer, both the real and simulated signals after correlation with the emitted code have been normalized.

Fig. 8 shows the real (top) and simulated (bottom) signals after correlation. Taking into account that a phase shift can provide a negative peak, the absolute value of the correlation signals, $|y(t)|$, is shown in Fig. 9 for a time window close to the first arrival. The arrivals whose amplitudes are bigger than the correlation noise have been remarked.

The first conclusion that can be obtained from this result is that the Kasami signal is clearly detected and the TOF can be easily obtained, although there is a considerable multipath component. This multipath can be noticed in Fig. 9 as the train of peaks that form the correlated signal. Since in this test the influence of three-dimensional propagation is not that important due to the dimensions of the pool and the directionality of the transducers, the propagation model obtains the TOF with good accuracy, as well as the relative amplitudes of the main peaks, such as that of the first arrival. The TOF can be obtained as the difference between the arrival times of the different paths and the duration of the emitted code. The TOF for the real and simulated signals are gathered into Table 1 , where only the first ten arrivals have been represented.

These times are similar for both signals: the difference in the values varies between 1 and $47 \mu \mathrm{s}$, when detected in both signals. As can be seen in Fig. 9, there is a first arrival followed by slightly

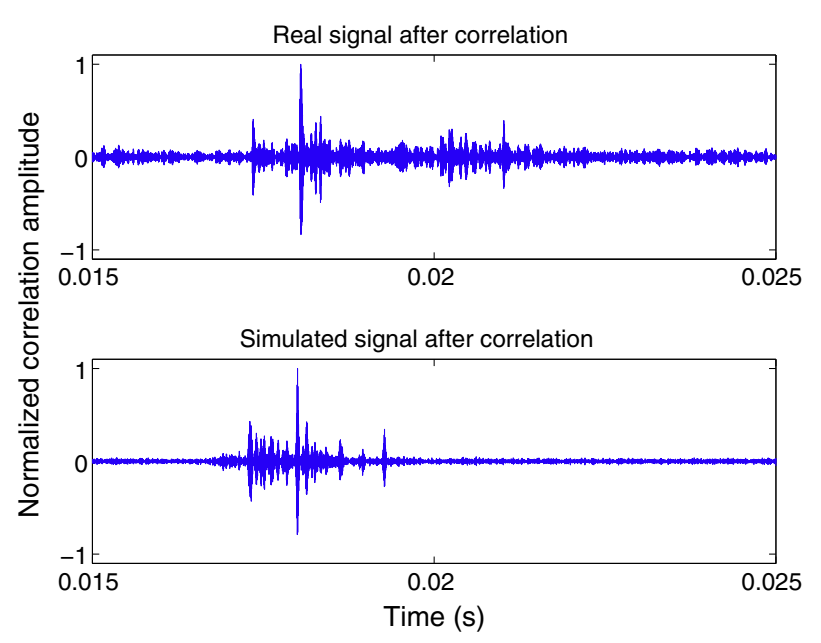

Fig. 8. Real and simulated signals after correlation in test one.

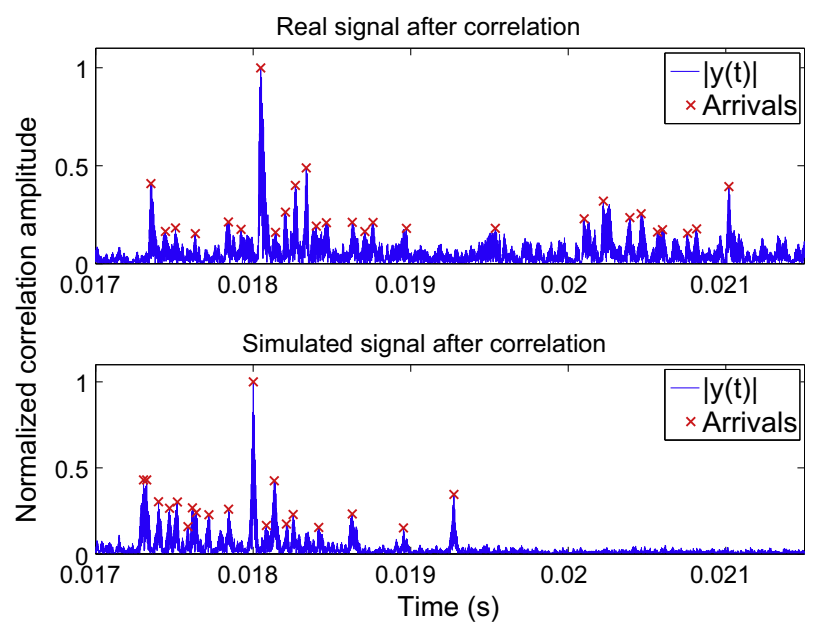

Fig. 9. Zoom of the absolute value $|y(t)|$ of the real and simulated signals after correlation in test one.

Table 1

Detected TOF for the real and simulated signals after correlation in test one.

\begin{tabular}{rcl}
\hline Path & TOF $_{\text {real }}(\mathrm{ms})$ & TOF $_{\text {sim }}(\mathrm{ms})$ \\
\hline 1 & & 16.674 \\
2 & 16.721 & 16.694 \\
3 & 16.813 & 16.769 \\
4 & & 16.839 \\
5 & 16.877 & 16.889 \\
6 & & 16.956 \\
7 & & 16.984 \\
8 & 17.003 & 17.009 \\
9 & & 17.089 \\
10 & 17.213 & 17.214 \\
\hline
\end{tabular}

weaker paths, and then the strongest one. This strongest path is followed again by weaker ones in a similar way, in both the real and simulated signals. This result indicates that the model is describing fairly well the main features of the underwater channel with regards to the multipath structure, TOF and even relative amplitude of the arrivals. Some minor differences appear in the interference region between the first arrival and the one with higher amplitude regarding the relative amplitudes, as well as in the paths coming later than the arrival with higher amplitude. From $20 \mathrm{~ms}$ onwards, some paths are detected in the real signal 


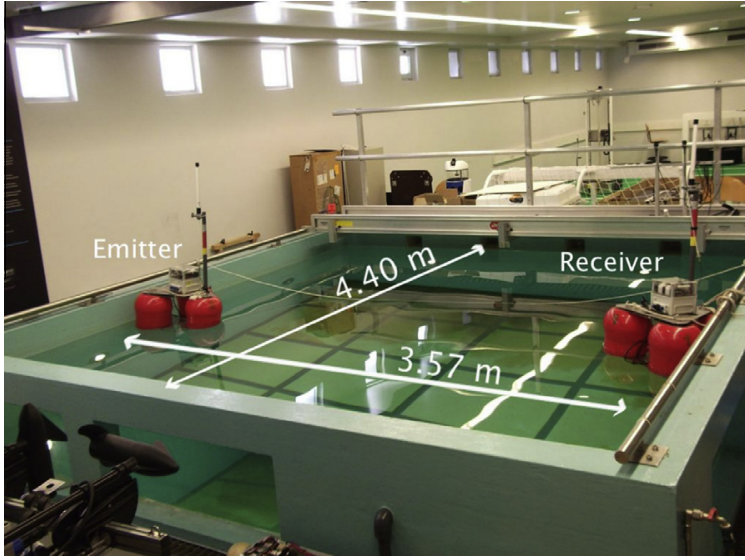

Fig. 10. Set up for test two.

coming from lateral rebounds in the pool that are not included in the propagation model. Nevertheless, the main features of the acoustic propagation are correctly predicted by the model.

\subsection{Test in pool two}

Considering the results obtained in the first pool, and since reverberation and multipath are strongly dependent on pool size, another test has been conducted in a smaller one, so the influence of reverberation and a very strong multipath component in the signal detection can be studied. The instrumentation employed in this test has also the advantage of a omnidirectional and easily synchronized transmission and reception of the acoustic signal, as well as the recording of the received signal in an internal memory for later processing.

The pool and its dimensions are shown in Fig. 10. This pool has a constant depth of $1.73 \mathrm{~m}$. Fig. 11(a) and (b) shows an example of ray-tracing performed with 20 rays for visual clarity, for the vertical and horizontal plane, respectively. The strong multipath effect that takes place in the pool can be noticed. Emitter and receiver are located at $0.53 \mathrm{~m}$ depth, and the rays are launched between $\pm 85^{\circ}$. For this test, both the emitter and receiver are T257 transducers from Neptune Sonar Ltd., that have a bandwidth of $16 \mathrm{kHz}$ and a center frequency of $25 \mathrm{kHz}$. The received signal is captured and stored in the FPGA using a sampling frequency of $106.582 \mathrm{kHz}$. The water temperature was measured on site, and it had a value of $21^{\circ} \mathrm{C}$. It is assumed to be constant in all the water column.

The emitted signal is a 63 bits Kasami code modulated in BPSK at $25 \mathrm{kHz}$ using three cycles per modulation symbol, for a total time length of $7.6 \mathrm{~ms}$ and a signal bandwidth of approximately $10 \mathrm{kHz}$. This bandwidth can be seen in Fig. 12, where the absolute value of the frequency spectrum, $|x(f)|$, is represented.

Fig. 13 shows the correlated signal with the Kasami code for the real signal (top) and the simulated signal (bottom). In order to obtain this simulated signal, the rays were launched between $-85^{\circ}$ and $85^{\circ}$ to replicate the characteristic emission of the transducer. A value of wind speed of $0 \mathrm{~m} \mathrm{~s}^{-1}, 30 \mathrm{~dB}$ of SNR and the dimensions that were defined in Fig. 10 were used in the simulation, along with the information of frequency response provided by the transducer manufacturer.

Since the signal is normalized at the receiver to store it at the FPGA memory, both the real and simulated correlated signals have been normalized. It can be seen that the simulated signal is shorter than the real one. This is due to the fact that the model does not consider all the rebounds on opposite sides of the pool, as well as the full three dimensional propagation, so there is less reverberation than in the real case, which in this pool is highly important.

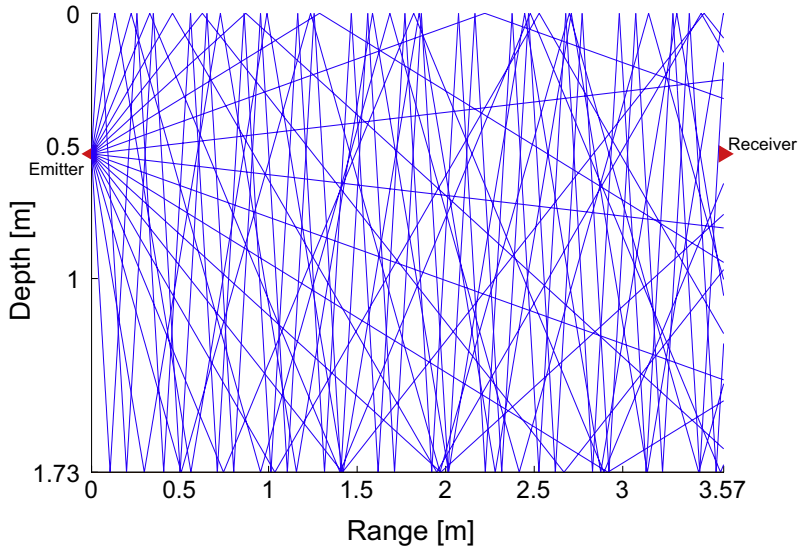

(a) Vertical plane

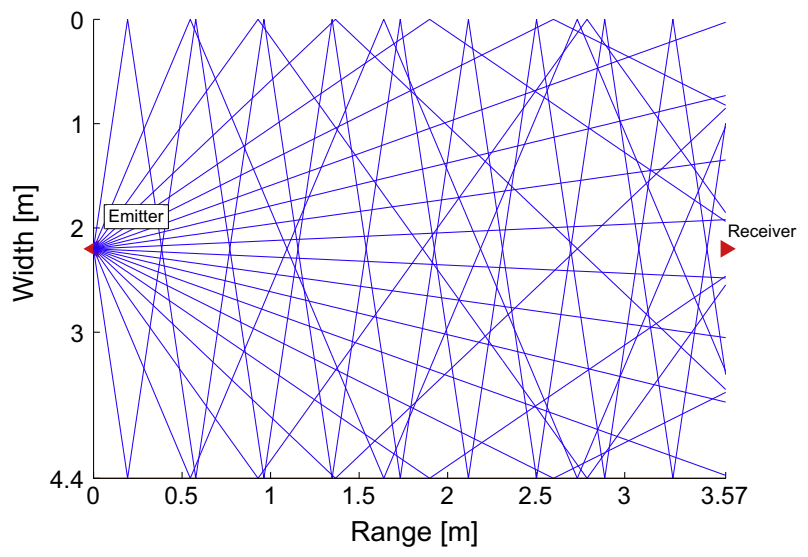

(b) Horizontal plane

Fig. 11. Ray-tracing for: (a) the vertical plane and (b) the horizontal plane in test two.

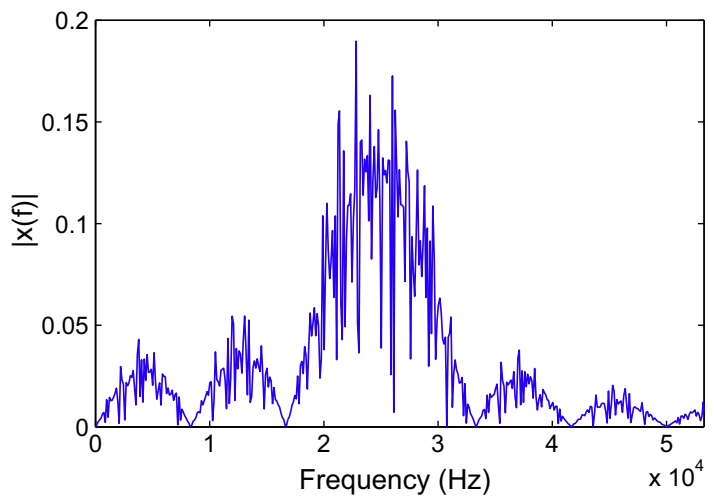

Fig. 12. Amplitude spectrum of the emitted signal in test two.

The absolute value for the correlation signal, $|y(t)|$, has been represented in Fig. 14 in a time window close to the first arrival, avoiding then much of the reverberation. The arrivals whose amplitudes are bigger than the correlation noise have been indicated.

Again, the TOF for the real and simulated signals are gathered into Table 2, where only the first ten arrivals have been represented.

The first conclusion that can be drawn is that the amplitudes of the detected arrivals are not exactly the same, especially for the third and fourth arrivals in the simulation. This disagreement 


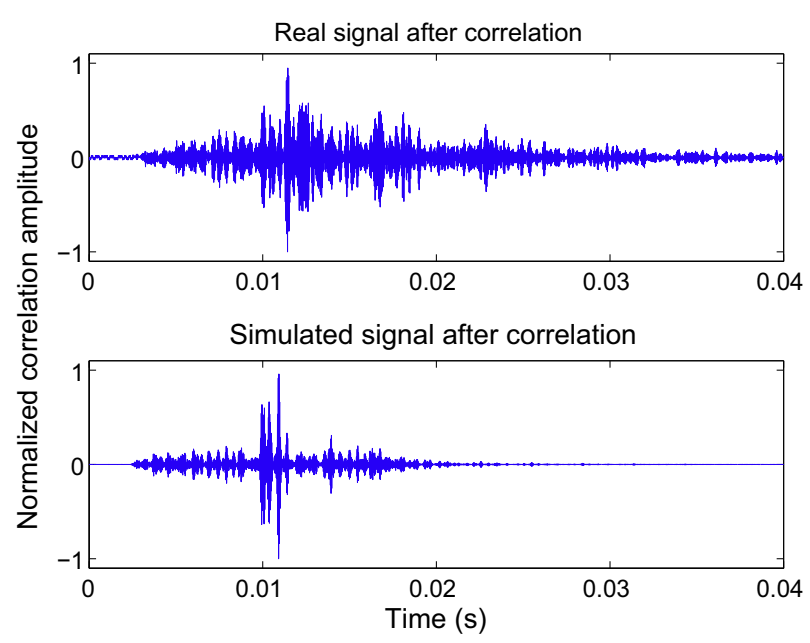

Fig. 13. Real and simulated signals after correlation, for test two.

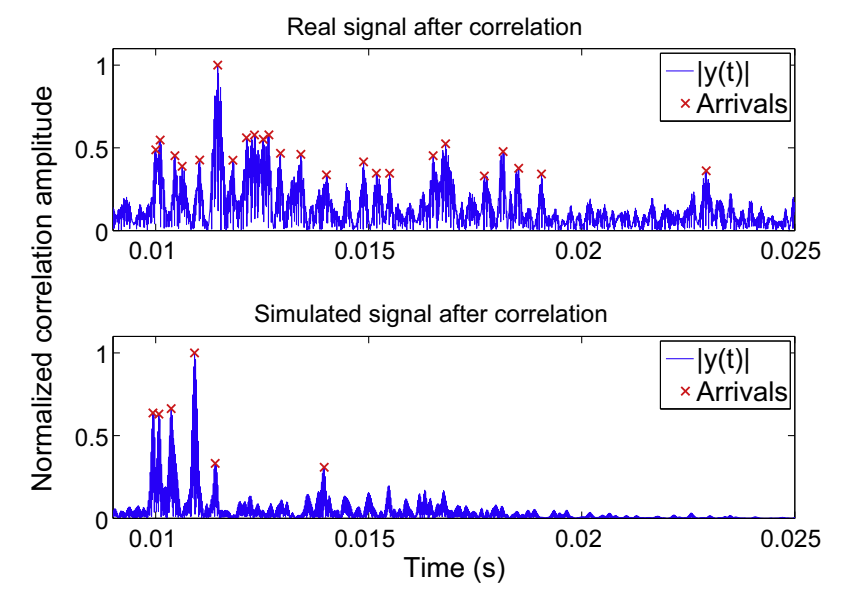

Fig. 14. Zoom of the absolute value $|y(t)|$ of the real and simulated signals after correlation, for test two.

Table 2

Detected TOF for the real and simulated signals after correlation in test two.

\begin{tabular}{cll}
\hline Path & TOF $_{\text {real }}(\mathrm{ms})$ & TOF $_{\text {sim }}(\mathrm{ms})$ \\
\hline 1 & 2.441 & 2.376 \\
2 & 2.544 & 2.516 \\
3 & 2.892 & 2.804 \\
4 & 3.060 & \\
5 & 3.473 & 3.352 \\
6 & 3.895 & 3.836 \\
7 & 4.252 & \\
8 & 4.571 & \\
9 & 4.759 & \\
10 & 4.965 & \\
\hline
\end{tabular}

comes mainly from the strong multipath and reverberation that is taking place, which is higher than in the first test. Since the emitter is omnidirectional and the dimensions of the pool are small, there will be a strong reverberation that is missed in the simulated signal. All these paths will start interfering tenths of milliseconds after the first arrivals. In this case, the artificially added paths are not enough to describe the behavior of the received signal starting few milliseconds after the direct path arrival, as can be seen in the several arrivals that are present in the real signal after correlation, but not in the simulated one.

Nevertheless, the signal is correctly detected even in this adverse environment as it is shown in Fig. 14, and the model detects the TOF fairly well for the first arrivals: from the first six arrivals in the real signal after correlation, the model predicts five of them, with errors ranging from $28 \mu$ s to $121 \mu \mathrm{s}$. In fact, for a system that only needs to detect the first arrival to work, such as a positioning system based on the detection of coded signals, the results show that it would detect the signal in this adverse scenario, since the arrivals are distinguishable.

\section{Summary and conclusions}

In this work, some experiments concerning the correct detection of underwater acoustic coded signals in strong multipath environments have been performed. In addition, these detections have been compared with the ones provided by a propagation model, in order to test the feasibility of the model for the prediction of propagation conditions and signal detections in other environments.

Two environments have been considered, representing underwater channels with different degrees of multipath and reverberation. In addition, different emitters have been tested, whose directionality allowed to modify the reverberation and multipath conditions. The emitted Kasami signal was correctly detected in both experiments, which show the robustness of this coding scheme to avoid detection errors in such adverse environments.

Additionally, these results have been compared with the ones provided by an underwater acoustic propagation model. Correlated signals obtained by simulations with the propagation model have been compared with the results obtained in pool tests, showing a similar behavior for the TOF in both experiments, specially when reverberation is not important. Even though the amplitudes were not exactly the same, the simulated results are closely related even for complicated scenarios with a strong multipath component, such as in test two for the first arrivals. The propagation paths are obtained with a time difference between 1 and $47 \mu$ s for test one, and between 28 and $122 \mu$ s for test two. These results indicate that the model describes fairly well the acoustic propagation that is taking place, so it could be used to study the propagation conditions and system performance even in such unfavorable environments.

\section{Acknowledgements}

This work has been possible thanks to the support of the Spanish Ministry of Economy and Competitiveness (LORIS project, ref: TIN2012-38080-C04-01/02) and the University of Alcalá (FPI fellowship). This work has also been financed by the ERDF - European Regional Development Fund through the COMPETE Programme (operational programme for competitiveness) and by National Funds through the FCT - Fundação para a Ciência e a Tecnologia (Portuguese Foundation for Science and Technology) within project "FCOMP-01-0124-FEDER-022701".

\section{References}

[1] Fischer JH, Bennett KR, Reible SA, Cafarella JH, Yao I. A high data rate, underwater acoustic data-communications transceiver. In: Proceedings of the OCEANS 1992 conference, vol. 2, Newport (RI), United States, October, 1992. p. 571-6.

[2] Haller DR, Lemon DD. Sidescan sonar detection performance with PRN coded signals. In: Proceedings of the OCEANS 1993 Conference, vol. 1, Victoria, Canada, October 1993. p. 237-42.

[3] Austin TC. The application of spread spectrum signaling techniques to underwater acoustic navigation. In: Proceedings of the 1994 symposium on autonomous underwater vehicle technology. Cambridge (MA), United States, July 1994. p. 443-9.

[4] Loubet G, Capellano V, Filipiak R. Underwater spread-spectrum communications. In: Proceedings of the OCEANS 1997 conference, vol. 1. Halifax, Canada, October 1997. p. 574-9. 
[5] Sozer EM, Proakis JG, Stojanovic M, Rice JA, Benson A, Hatch M. Direct sequence spread spectrum based modem for under water acoustic communication and channel measurements. In: Proceedings of the 1999 OCEANS conference, vol. 1. Seattle (WA), United States, September 1999. p. 228-33.

[6] Frassati F, Lafon C, Laurent P, Passerieux J. Experimental assessment of OFDM and DSSS modulations for use in littoral waters underwater acoustic communications. In: Proceedings of the OCEANS 2005 Europe Conference vol. 2. Brest, France, June 2005. p. 826-31.

[7] Zhang G, Hovem JM, Dong H, Liu L. Experimental studies of underwater acoustic communications over multipath channels. In: Conference publications of the fourth international conference on sensor technologies and applications. Venice, Italy, July 2010. p. 458-61.

[8] Etter PC. Underwater acoustic modeling and simulation. 3rd ed. New York (NY), United States: Taylor and Francis; 2003.

[9] Buckingham MJ. Ocean-acoustic propagation models. J Acoust 1992:223-87.

[10] Weinberg H, Keenan RE. Gaussian ray bundles for modeling high-frequency propagation loss under shallow-water conditions. Naval Undersea Warfare Center Division, Newport, RI, United States, NUWC-NPT Technical Report 10,568, April 1996.

[11] Bowlin JB, Spiesberger JL, Duda TF, Freitag LF. Ocean acoustical ray-tracing software RAY. Woods Hole Oceanographic Institution, Woods Hole, MA, United States, Technical Report WHOI-93-10, October 1992.

[12] Porter MB. The BELLHOP manual and user's guide: Preliminary draft <http:/ oalib.hlsresearch.com/Rays/HLS-2010-1.pdf>, 2014 [accessed 18.03.14].

[13] Bjerrum-Niese C, Lützen R. Stochastic simulation of acoustic communication in turbulent shallow water. IEEE J Ocean Eng 2000:25(4):523-32.

[14] Chitre M. A high-frequency warm shallow water acoustic communications channel model and measurements. J Acoust Soc Am 2007;122(5):2580-6.
[15] Kinsler LE, Frey AR, Coppens AB, Sanders JV. Fundamentals of acoustics, 4th ed. New York (NY), United States: John Wiley \& Sons; 2000.

[16] National Physical Laboratory. Technical guides: speed of sound in sea-water <http://resource.npl.co.uk/acoustics/techguides/soundseawater/> [accessed 18.03.14].

[17] Leroy CC, Parthiot F. Depth-pressure relationships in the oceans and seas. J Acoust Soc Am 1998;103(3):1346-52.

[18] Urick RJ. Principles of underwater sound. 3rd ed. Los Altos Hills (CA), United States: Peninsula Publishing: 1983.

[19] Francois RE, Garrison GR. Sound absorption based on ocean measurements: part I: pure water and magnesium sulfate contributions. J Acoust Soc Am 1982;72(3):896-907.

[20] Francois RE, Garrison GR. Sound absorption based on ocean measurements: part II: boric acid contribution and equation for total absorption. J Acoust Soc Am 1982;72(6):1879-90.

[21] Brekhovskikh LM, Lysanov LP. Fundamentals of ocean acoustics. 3rd ed. New York (NY), United States: Springer; 2003.

[22] Coates R. An empirical formula for computing the Beckman-Spizzichino surface reflection loss coefficient. IEEE Trans Ultrason, Ferroelectr, Freq Control 1988;35(4):522-3.

[23] Stojanovic M. Wiley encyclopedia of telecommunications. In: Proakis JG, editor. Ch. acoustic (underwater) communications, vol. 1. New York (NY), United States: John Wiley \& Sons; 2003. p. 36-47.

[24] Kasami T. Weight distribution formula for some class of cyclic codes, Coordinated Science Lab., University of Illinois, Urbana, IL, United States, Technical Report R-285, April 1966. 